\title{
Influence of premetallization surface treatment on the formation of Schottky Au-nGaN contacts
}

T. G. G. Maffeis, M. C. Simmonds, and S. A. Clark

Materials Research Institute, Sheffield Hallam University, Sheffield, S1 1WB, United Kingdom

F. Peiro

Department of Electronics, University of Barcelona, Marti i Franques 1, 08028 Barcelona, Spain

P. Haines and P. J. Parbrook

Department of Electronic and Electrical Engineering, University of Sheffield, Sheffield, S1 3JD, United Kingdom

(Received 22 March 2002; accepted for publication 27 June 2002)

\begin{abstract}
The influence of premetallization surface preparation on the structural, chemical, and electrical properties of $\mathrm{Au}-n \mathrm{GaN}$ interfaces has been investigated by x-ray photoemission spectroscopy (XPS), current-voltage measurement $(I-V)$ and cross-section transmission electron microscopy (TEM). XPS analysis showed that the three GaN substrate treatments investigated i.e., ex situ hydrofluoric acid etch, in situ anneal in ultrahigh-vacuum (UHV), and in situ Ga reflux cleaning in UHV result in surfaces increasingly free of oxygen contamination. XPS and TEM characterization of $\mathrm{Au}-n \mathrm{GaN}$ formed after the three premetallization surface treatments show that HF etching and UHV annealing produce abrupt, well-defined interfaces. Conversely, $\mathrm{GaN}$ substrate cleaning in a $\mathrm{Ga}$ flux results in $\mathrm{Au} / \mathrm{GaN}$ intermixing. $I-V$ characterization of $\mathrm{Au}-n \mathrm{GaN}$ contacts yields a Schottky barrier height of $1.25 \mathrm{eV}$ with a very low-ideality factor and very good contact uniformity for the premetallization UHV anneal, while the Ga reflux cleaning results in a much lower barrier $(0.85$ $\mathrm{eV}$ ), with poor ideality and uniformity. $I-V$ and XPS results suggest a high density of acceptor states at the surface, which is further enhanced by UHV annealing. These results are discussed in the context of current models of Schottky barrier formation. (c) 2002 American Institute of Physics.
\end{abstract}

[DOI: $10.1063 / 1.1501750]$

\section{INTRODUCTION}

Gold is one of the most important metals in semiconductor device fabrication because of its excellent electrical conductivity and its resistance to oxidation. Additionally, its high work function $(5.2 \mathrm{eV})$ means gold is an ideal choice for Schottky rectifiers on $n \mathrm{GaN}$ and for Ohmic contacts to $p \mathrm{GaN}$. Au-GaN contact formation has been widely investigated $^{1-7}$ by current-voltage $(I-V)$ and capacitance measurement techniques. These works report that gold does indeed form rectifying contacts on $n \mathrm{GaN}$, with measured barrier heights ranging from 0.80 to $1.1 \mathrm{eV}$. This wide range of values is thought to be a consequence of different growth techniques and parameters, surface polarity, and/or surface treatment.

Koyama et al. ${ }^{8}$ reported the influence of predeposition surface treatment on the electrical characteristics of Au contacts on $n \mathrm{GaN}$. They found that diodes formed after cleaning in organic solvents exhibited high leakage currents and poor ideality factors, while those formed after a hydrofluoric acid $(\mathrm{HF}) / \mathrm{HCl}$ treatment were nearly Ohmic. On the other hand, contacts thermally deposited after cleaning in a warm $\mathrm{NH}_{4} \mathrm{OH}$ solution or electro-deposited exhibited near ideal Schottky behavior. This study, as well as the wide range of values reported for $\mathrm{Au}-n \mathrm{GaN}$ Schottky barrier heights, highlights the relevance of a systematic investigation of the influence of predeposition surface treatment.
Furthermore, only two groups ${ }^{9,10}$ have reported $\mathrm{x}$-ray photoemission spectroscopy (XPS) studies of Au deposition on atomically clean $n \mathrm{GaN}$, and while the XPS measured barrier heights are similar in both cases, the proposed mechanisms by which this barrier is reached differ widely. Wu and $\mathrm{Kahn}^{10}$ found that their $1.2 \mathrm{eV}$ barrier was due to an initial upward band bending at the bare $\mathrm{GaN}$ surface of $+0.7 \mathrm{eV}$ and to a further $+0.5 \mathrm{eV}$, as a result of Au-induced band bending. Sporken et al. ${ }^{9}$ reported a bare surface band bending of $+2.2 \mathrm{eV}$ and $\mathrm{a}-1.05 \mathrm{eV}$ downward band bending following Au deposition.

The divergence between the two experiments clearly calls for further XPS investigations in order to clarify the $\mathrm{Au}-n \mathrm{GaN}$ contact formation mechanisms. Furthermore, the effect of surface preparation prior to metallization has not yet been investigated by XPS. In this article, we compare the XPS, current-voltage $(I-V)$, and transmission electron microscopy (TEM) characterization of Au contacts deposited on $n \mathrm{GaN}$ after three different surface treatments: (i) ex situ chemical cleaning in HF solution, (ii) in situ annealing, and (iii) in situ cleaning by the Ga deposition, reduction, and reevaporation technique. ${ }^{11-13}$

\section{EXPERIMENTAL DETAILS}

The samples used in all three experiments were cut from the same $1.1 \mu \mathrm{m}$ thick $\mathrm{GaN}$ epilayer, grown at $1020^{\circ} \mathrm{C}$ by metal-organic vapor phase epilaxy (MOVPE) on sapphire 
TABLE I. Sequence of Au depositions.

\begin{tabular}{lllllllllll}
\hline \hline $\begin{array}{l}\text { Incremental deposition } \\
\text { thickness }(\AA)\end{array}$ & 0.5 & 0.5 & 1 & 2 & 3 & 5 & 8 & 15 & 20 & 1500 \\
\hline Total coverage $(\AA)$ & 0.5 & 1 & 2 & 4 & 7 & 12 & 20 & 35 & 55 & 2000 \\
\hline \hline
\end{tabular}

along the $c$ axis. Hall measurements yielded a carrier concentration of $2 \times 10^{15} \mathrm{~cm}^{-3}$ and a mobility of 41 $\mathrm{cm}^{2} \mathrm{~V}^{-1} \mathrm{~s}^{-1}$ at room temperature. The three surface preparations were as follows:

\section{(a) Ex situ chemical etch}

Prior to loading in ultrahigh vacuum (UHV), the sample was degreased in acetone, dipped in HF: de-ionized (DI) $\mathrm{H}_{2} \mathrm{O}(1: 10)$ for $1 \mathrm{~min}$, and rinsed in DI water.

(b) In situ annealing

This sample was first subjected to the same ex situ treatment as in (a). Following loading in UHV, the sample was additionally annealed at $600{ }^{\circ} \mathrm{C}$ for $10 \mathrm{~min}$. The sample temperature was monitored with an optical pyrometer.

(c) In situ Ga reflux cleaning

This sample was first subjected to the same ex situ treatment as in (a). Following loading in UHV, the sample was then subjected to two cycles of the Ga deposition, reduction, and reevaporation cleaning method. This method, also known as Ga reflux cleaning, involves evaporating Ga metal onto the GaN substrate, to react with oxygen contaminants. The substrate was then heated up to $900{ }^{\circ} \mathrm{C}$ in order to desorb both $\mathrm{Ga}$ metal atoms and oxygen, leaving a clean $\mathrm{GaN}$ surface.

After each of the three surface preparations, Au was deposited in steps of increasing thickness from submonolayer coverages to several monolayers, as shown in Table I. The thickness was measured by a quartz crystal monitor. XPS scans of the $\mathrm{Ga} 3 d, \mathrm{Ga} 2 p_{3 / 2}, \mathrm{~N} 1 s$, and $\mathrm{Au} 4 f$ core levels were recorded at normal emission and $60^{\circ}$ off normal emission before and after every deposition. A thick layer of $\mathrm{Au}$ was deposited at the end of the experiment to facilitate the processing of the samples into diodes for $I-V$ characterization. The samples were patterned by conventional lithography with circular dots of diameters of $0.17,0.22,0.44,0.64$, and $0.84 \mu \mathrm{m}$. A KI:DI $\mathrm{H}_{2} \mathrm{O}$ solution was used to etch the $\mathrm{Au}$ and expose the underlying GaN surface. The Ohmic contact

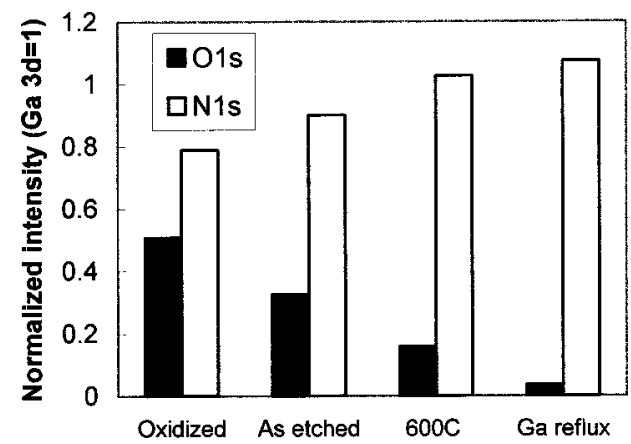

FIG. 1. Normalized XPS core-level intensity from the oxidized, HF etched, annealed, and $\mathrm{Ga}$ reflux cleaned surfaces. The intensities have been weighted by an empirical sensitivity factor and normalized to the $\mathrm{Ga} 3 d$ intensity. was provided by a large-area Au contact left on the sample during lithography. ${ }^{2,14,15}$ The XPS spectra were acquired using a VG Microlab system attached to a custom-built evaporation chamber. The base pressure was $10^{-10} \mathrm{mb}$ and the $\mathrm{Mg}$ anode gave an energy resolution of $0.9 \mathrm{eV}$. The cross-section TEM observation were carried out on a Hitachi H800-NA electron microscope operating at $200 \mathrm{kV}$.

\section{EXPERIMENTAL RESULTS}

\section{A. Core-level intensity}

Prior to $\mathrm{Au}$ deposition, the three surfaces were checked for oxygen contamination. Figure 1 shows the $\mathrm{O} 1 s$ to Ga $3 d$ and $\mathrm{N} 1 s$ to $\mathrm{Ga} 3 d$ core-level intensity ratios for the three surfaces under investigation, compared to the oxidized ("as received") surface. The amount of oxygen contamination is clearly reduced by each of the three, increasingly complex, surface preparations. The oxygen peak was barely detectable by XPS after cleaning with the Ga reflux method. Additionally, the $\mathrm{N} 1 s$ to $\mathrm{Ga} 3 d$ intensity ratio increases from 0.8 for the oxidized surface to 1 after UHV annealing, indicating that the surface has become stochiometric (within the sensitivity of XPS).

The attenuation of the Ga $3 d$, Ga $2 p_{3 / 2}$, and $\mathrm{N} 1 s$ corelevel intensities plots for the $600{ }^{\circ} \mathrm{C}$ annealed surface, as a function of $\mathrm{Au}$ coverage, are displayed in Fig. 2. As ex-
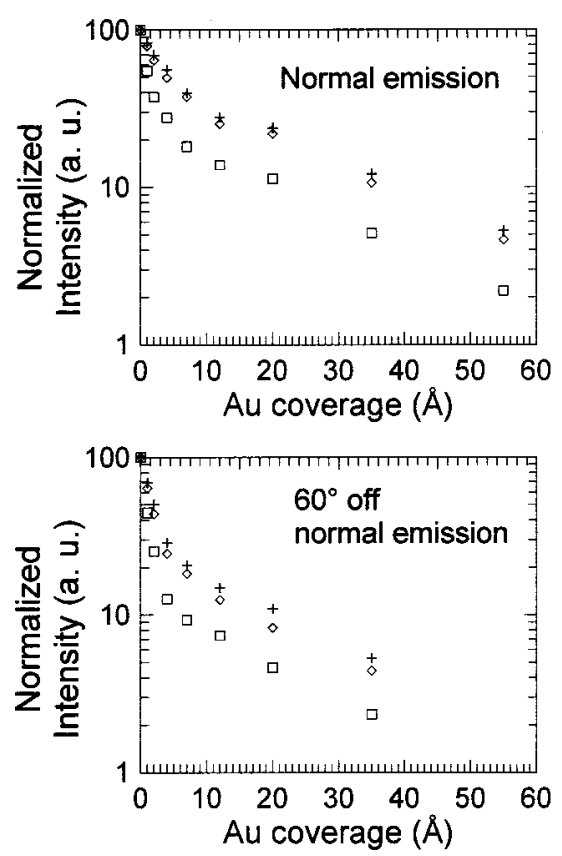

FIG. 2. Ga $3 d$ (crosses), N $1 s$ (diamond shapes), and Ga $2 p_{3 / 2}$ (squares) integrated core-level intensity as a function of Au coverage at normal emission and $60^{\circ}$ off normal emission for the annealed surface. The data points are normalized to the intensity at $0 \AA$ Au coverage. 
TABLE II. Photoelectron mean free paths of the Ga $3 d$ core level for the three different surface treatments at normal and $60^{\circ}$ off normal emission ( $45^{\circ}$ off for the HF etched surface), compared to the value calculated using Eq. (1).

\begin{tabular}{lccc}
\hline \hline & \multicolumn{3}{c}{ Mean free path $(\AA)$} \\
\cline { 2 - 4 } Ga $3 d$ & Normal emission & $60^{\circ}$ off & $45^{\circ}$ off \\
\hline Theoretical value & 19.0 & 9.5 & 13.5 \\
$600{ }^{\circ} \mathrm{C}$ anneal & 5.4 & 2.6 & \\
Ga reflex & 2.8 & 2.8 & \multirow{2}{*}{8.0} \\
HF etch & 10.0 & & \\
\hline \hline
\end{tabular}

pected, the intensity drop with Au coverage is sharper at $60^{\circ}$ off normal emission because of the shorter photoelectron escape depth. Similarly, the Ga $2 p_{3 / 2}$ core-level intensity drops faster than the $\mathrm{Ga} 3 d$ or $\mathrm{N} 1 s$ because of the difference in photoelectron kinetic energy, which makes the Ga $2 p_{3 / 2}$ core level more surface sensitive.

The escape depth, or photoelectron mean-free-path (MFP) $\lambda$ can be calculated from the atomic mass $A$ of the element, the bulk material density $\rho$, the Avogadro number $N$, and the photoelectron kinetic-energy $E_{\text {kin }}$ with the following equation: ${ }^{16}$

$$
\lambda(\mathrm{nm})=0.41\left[\frac{A\left(\mathrm{~g} \mathrm{~mol}^{-1}\right) 10^{24}}{\rho\left(\mathrm{kg} \mathrm{m}^{-3}\right) N\left(\mathrm{~mol}^{-1}\right)} E_{\mathrm{km}}(\mathrm{eV})\right]^{1 / 2} .
$$

Layer-by-layer growth leads to exponential decay of the XPS core-level intensity $I$, from the substrate as the metal overlayer thickness $z$ increases

$$
I \propto \exp \left(-\frac{z}{\lambda}\right),
$$

where $\lambda$ is the attenuation length, or the MFP. Both graphs in Fig. 2 clearly show two attenuation regimes with increasing Au deposition: below $5 \AA \mathrm{Au}$ coverage, the intensity falls rapidly and exponentially; while above $10 \AA$, the attenuation is less steep. This behavior is indicative of layer-by-layer (or two-dimensional) growth followed by islanding (Stransky-Krastanov growth). ${ }^{17}$ This is, to some extent, in contradiction with the results reported by Sporken et al. ${ }^{9}$ who found that $\mathrm{Au}$ growth is purely two dimensional on $n \mathrm{GaN}$ at room temperature, the precise origin of this discrepancy remains an open question.

Fitting Eq. (2) to the experimental data points from the initial stages of Au deposition therefore yields an experimental estimate of the MFP calculated with Eq. (1). The comparison of experimental and theoretical values for the $\mathrm{Ga} 3 d$ and $\mathrm{N} 1 s$ core levels is given in Tables II and III, respectively. First, these tables confirm the trend shown in Fig. 2, i.e., the MFP at $60^{\circ}$ off normal emission is half that of normal emission for the $600{ }^{\circ} \mathrm{C}$ annealed surface. The same is also true for the HF etched surface where the ratios of experimental MFP's at $45^{\circ}$ off normal emission and normal emission are close to the theoretical value of 1.414 . In the case of the Ga reflux surface, however, the MFP at $60^{\circ}$ off normal emission is similar (even slightly larger for the $\mathrm{N} 1 s$ core level) to the normal emission MFP. This anomaly seems to indicate that the $\mathrm{Au}-\mathrm{GaN}$ interface is not abrupt, possibly
TABLE III. Photoelectron mean free paths of the N $1 s$ core level for the three different surface treatments at normal and $60^{\circ}$ off normal emission ( $45^{\circ}$ off for the HF etched surface), compared to the value calculated using Eq. (1).

\begin{tabular}{lccc}
\hline \hline & \multicolumn{3}{c}{ Mean free path $(\AA)$} \\
\cline { 2 - 4 } $\mathrm{N} 1 s$ & Normal emission & $60^{\circ}$ off & $45^{\circ}$ off \\
\hline Theoretical value & 16.0 & 8.0 & 11.0 \\
$600{ }^{\circ} \mathrm{C}$ anneal & 4.3 & 2.4 & \\
Ga reflux & 2.2 & 2.8 & \multirow{2}{*}{5.9} \\
HF etch & 9.1 & & \\
\hline \hline
\end{tabular}

as a result of chemical reactions, Au in-diffusion, or surface roughening caused by the Ga reflux cleaning. Alternatively, the assumption of Stransky-Krastanov growth might not be justified, in which case Au growth would be purely three dimensional. The central point, however, is that attenuation plots for the Ga reflux cleaned surface display fundamental differences from the well-behaved $600{ }^{\circ} \mathrm{C}$ annealed and $\mathrm{HF}$ etched surfaces.

\section{B. Binding-energy shift}

Figure 3 shows the evolution of the Ga $3 d$ core-level peak shape for the annealed surface with incremental $\mathrm{Au}$ coverage. The stack plot also shows the effect of the $600{ }^{\circ} \mathrm{C}$ anneal on the HF etched surface (bottom two curves). The evolution of the binding energy of the Ga $3 d$ and N $1 s$ core levels for the three surfaces with in situ cleaning procedure and increasing Au coverage is summarized in Figs. 4 and 5. It is apparent that, for a given surface preparation, both $\mathrm{GaN}$

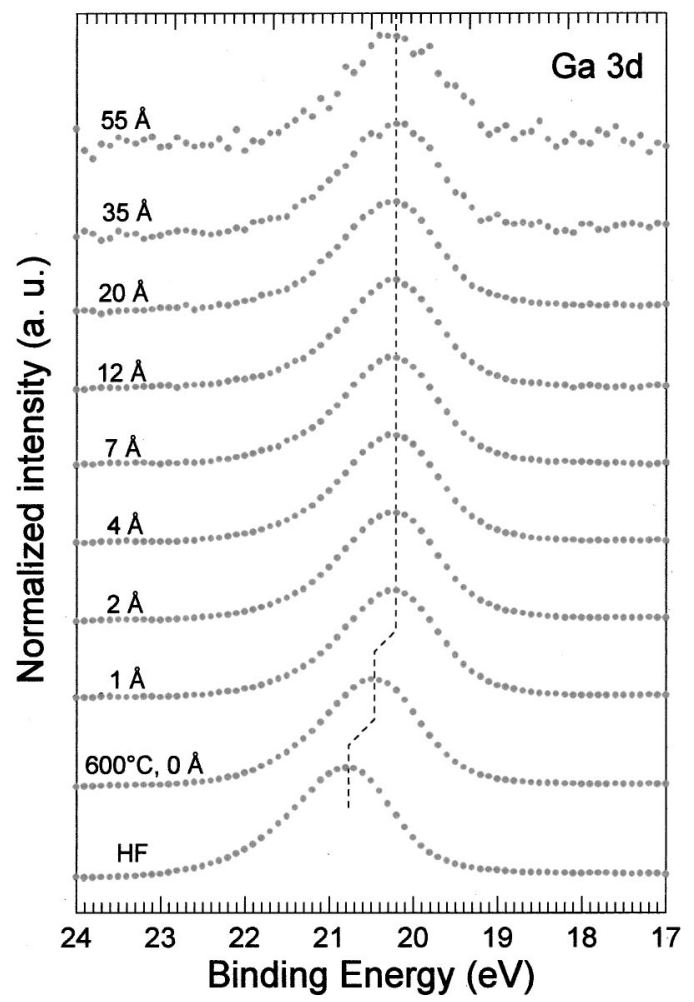

FIG. 3. Evolution of the Ga $3 d$ core level following in situ annealing and incremental Au deposition. The dashed line is intended as a guide to the eye. 


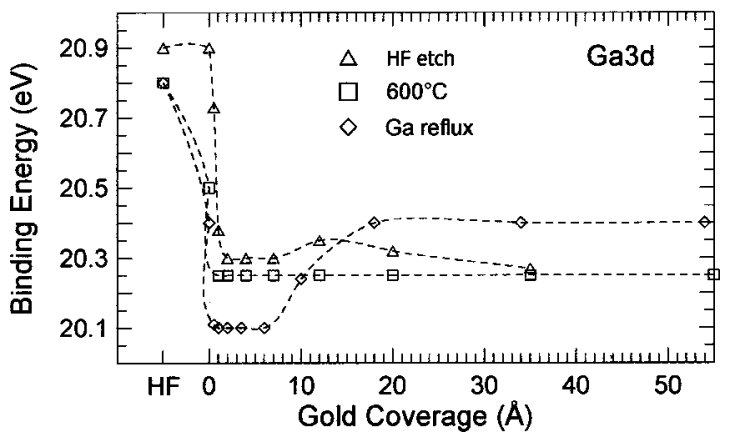

FIG. 4. Variation of the Ga $3 d$ core-level binding energy with surface preparation and increasing $\mathrm{Au}$ coverage for the three surfaces. Dashed lines are intended as a guide to the eye.

core levels follow the same trend. Additionally, curve fitting of the Ga $3 d$ and N $1 s$ spectra (not shown) reveals no evidence of chemical shift. Therefore, it is likely that the binding-energy shifts are rigid Fermi shifts and correspond to band bending at the interface.

Both in situ cleaning techniques of the HF etched surface resulted in upward band bending, of $0.35 \mathrm{eV}$ for the $600{ }^{\circ} \mathrm{C}$ annealed surface, and $0.45 \mathrm{eV}$ for the Ga reflux surface. This is consistent with previous work, which reports upward band bending as a result of UHV annealing. ${ }^{18}$

Figures 4 and 5 show that following Au deposition, the $600{ }^{\circ} \mathrm{C}$ annealed surface and the HF etched surface followed the same trend: the first few Au depositions (up to $2 \AA$ total coverage) induced upward band bending and subsequent $\mathrm{Au}$ depositions did not induce any further band bending, indicating that charge transfer was complete at this stage. Au deposition induced a band bending of $0.6 \mathrm{eV}$ on the HF etched surface. The total band bending following deposition of $\mathrm{Au}$ on the annealed surface was also $0.6 \mathrm{eV}$; comprised of the $0.35 \mathrm{eV}$ shift on annealing and a further $0.25 \mathrm{eV}$ shift on $\mathrm{Au}$ deposition. The Ga reflux surface also exhibited a slight upward band bending after the initial $\mathrm{Au}$ depositions but the bands bent back down at higher Au coverage, so that the Au-induced band bending is $0.0 \mathrm{eV}$. The total upward band bending for the Ga reflux surface, combining in situ cleaning and $\mathrm{Au}$ deposition is therefore $0.45 \mathrm{eV}$.

Figure 6 shows the evolution of the binding energy of the $\mathrm{Au} 4 f_{7 / 2}$ core level as a function of Au coverage for the

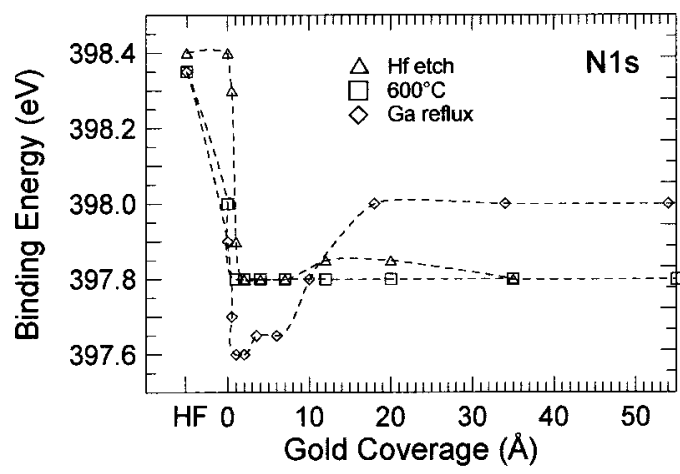

FIG. 5. Variation of the N $1 s$ core-level binding energy with surface preparation and increasing $\mathrm{Au}$ coverage for the three surfaces. Dashed lines are intended as a guide to the eye.

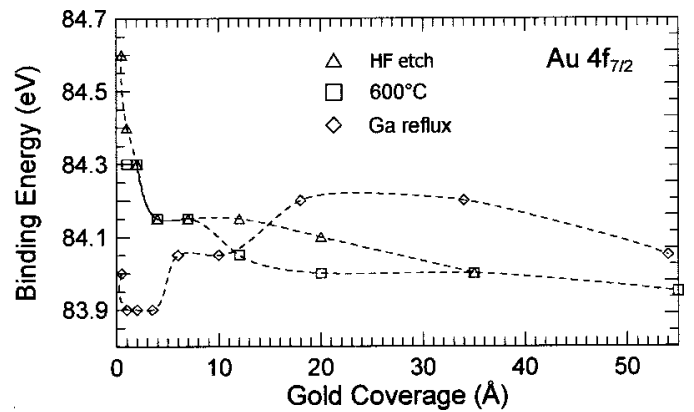

FIG. 6. Variation of the Au $4 f_{7 / 2}$ core-level binding energy with increasing $\mathrm{Au}$ coverage for the three surfaces. Dashed lines are intended as a guide to the eye.

three surface preparations. Again, the annealed and etched surfaces display very similar shifts while the Ga reflux surface behaves in a very different way. For the two former surfaces, the binding energy decreases from $84.6 \mathrm{eV}$ at $0.5 \AA$ to $84 \mathrm{eV}$ at $55 \AA .84 \mathrm{eV}$ is the binding energy of clean, metallic $\mathrm{Au}$, used to calibrate the instrument before the experiment. Since the Au peak is significantly broader at low coverage, the shift is likely to be related to changes in the chemical state of Au. This has been observed before by Sporken et al. ${ }^{9}$ who linked this broadening to a high-binding energy additional component attributed to $\mathrm{Ga}-\mathrm{Au}$ bonding. They reported that this component probably corresponded to only one atomic plane, indicating an abrupt interface. The $\mathrm{Au}$ $4 f_{7 / 2}$ core-level binding energies of $\mathrm{Ga}-\mathrm{Au}$ alloys have been measured using XPS by Jayne et al. ${ }^{19}$ Crucially, they found that the Ga $3 d$ binding energy shifted less than $0.3 \mathrm{eV}$ over the whole range of alloy compositions whilst the $\mathrm{Au} 4 f_{7 / 2}$ binding energy spanned over $1.5 \mathrm{eV}$. This could explain why no chemical shift was observed on the Ga $3 d$ scans while the $\mathrm{Au} 4 f_{7 / 2}$ core level shifted $0.6 \mathrm{eV}$ as the Au coverage increases. The interface is therefore likely to consist of a thin $\mathrm{Au}-\mathrm{Ga}$ alloy layer, which is rapidly buried under pure $\mathrm{Au}$.

By contrast, the Ga reflux surface presents strong differences. The binding-energy position of the $\mathrm{Au} 4 f_{7 / 2}$ peak is 84 $\mathrm{eV}$ after the first $\mathrm{Au}$ deposition. The peak position then moves slightly to lower-binding energy at low-Au coverage (1 to $3.5 \AA$ ) and to higher-binding energy at high-Au coverage ( 10 to $34 \AA$ ) but is back to $84 \mathrm{eV}$ after the last deposition (54 ̊). This indicates a fundamentally different interface formation mechanism, in which chemical changes occurred within a thick interfacial layer.

\section{I- $V$ measurements}

Figure 7 shows typical current versus voltage characteristics $(I-V)$ of the $\mathrm{Au}-n \mathrm{GaN}$ diodes fabricated on the (a) $600{ }^{\circ} \mathrm{C}$ annealed and (b) Ga reflux cleaned surfaces. The $I-V$ curve from the $600^{\circ} \mathrm{C}$ annealed surface exhibits a large forward bias threshold current and nondetectable reverse bias current indicative of a high barrier. By contrast, the $I-V$ curves measured on diodes formed after cleaning with the Ga reflux method show a much lower forward bias threshold and a high reverse bias current. Figure 7(b) also shows the influence of white light illumination while recording the currentvoltage characteristics. The reverse current of the measure- 

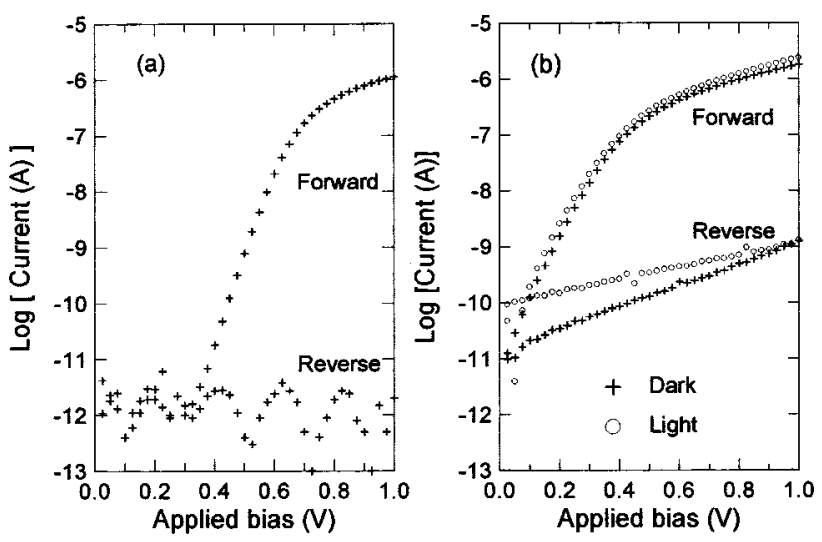

FIG. 7. Semilog plots of the current-voltage characteristics of typical diodes fabricated on (a) the $600{ }^{\circ} \mathrm{C}$ annealed surface and (b) the Ga reflux cleaned surface. Crosses designate measurement conducted in the dark while open circles refer to illuminated measurements.

ment under illumination at low reverse bias is an order of magnitude higher than that of the measurement in the dark. The forward current is also higher in the illuminated case. The ideality factors for the illuminated and dark measurements were 1.66 and 1.61 , for a barrier height of 0.87 and $0.88 \mathrm{eV}$, respectively. This behavior could be related to lightenhanced surface generation-recombination current contributions. Even though the $\mathrm{GaN}$ layer should be transparent to white light because of its $3.4 \mathrm{eV}$ band gap, it is possible that deep states within the band gap could act as generationrecombination centers. The fact that the sample glowed white under x-ray illumination during the XPS experiment seems to confirm the presence of radiative recombination in the visible range of the electromagnetic spectra. Brillson et $a l .{ }^{20}$ detected the presence of several deep states at native $\mathrm{GaN}$ surfaces using low-energy electron-excited nanoscale luminescence spectroscopy. They also found that states are created after annealing at high temperature in UHV or after metal deposition. Additionally, deep level transient spectroscopy measurement of Au contacts ${ }^{21}$ fabricated by thermal evaporation on MOVPE-grown $n \mathrm{GaN}$ revealed two defects at energies 0.27 and $0.61 \mathrm{eV}$ below the conduction-band minimum.

The Schottky parameters (zero-bias barrier height and ideality factor) were extracted by fitting the exponential section of the forward bias current by

$$
I=I_{0} \exp \left(\frac{q V}{n k T}\right),
$$

where $q$ is the electron charge, $V$ is the applied bias, $n$ the ideality factor, $k$ the Boltzmann constant, $T$ the temperature, and $I_{0}$ is given by

$$
I_{0}=S A^{* *} T^{2} \exp \left(-\frac{q \phi_{b 0}}{k T}\right)
$$

with $S, A^{* *}$ and $\phi_{b 0}$, the contact area, the Richardson constant (the theoretical value ${ }^{3}$ of $A^{* *}=26 \mathrm{~A} \mathrm{~cm}^{-2} \mathrm{~K}^{-2}$ was used) and the barrier height at zero bias, respectively.

The barrier heights thus extracted are plotted as a function of the ideality factor for a number of diodes fabricated

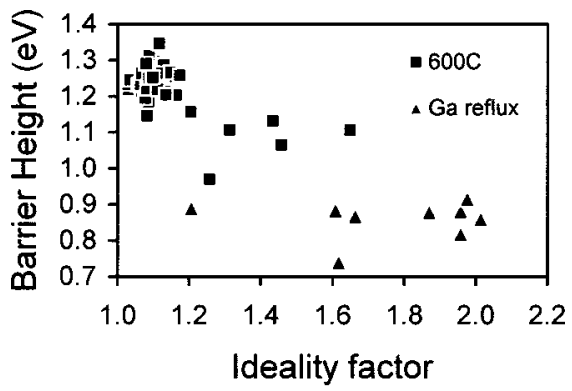

FIG. 8. Barrier height vs ideality factor for $\mathrm{Au}-n \mathrm{GaN}$ diodes formed on two different surfaces. The Schottky parameters have been extracted by the conventional method.

on the two surfaces in Fig. 8. The annealed surface resulted in diodes with low-ideality factors (mean $n=1.10$ and best $n=1.03$ ) and high barrier (mean $\phi_{b 0}=1.24 \mathrm{eV}$, highest $\phi_{b 0}=1.35 \mathrm{eV}$ ). The small spread of data points indicates good contact uniformity across the sample. On the other hand, only 9 (out of more than 50 measured) diodes fabricated on the Ga reflux cleaned surface had ideality factors below $n=2$ and some diodes exhibited Ohmic-like characteristics. The departure from ideality could be attributed to both recombination and tunneling effects which might be due to point surface defects caused by threading dislocations acting as recombination and tunneling centers. Because of the high series resistances, the $I-V$ curves from both surfaces were also analyzed using the small conductance method proposed by Werner et al.,22 which yielded similar results. ${ }^{15}$

\section{TEM results}

In an attempt to gain a better understanding of the $\mathrm{Au}-$ $\mathrm{GaN}$ interfaces, the samples were also investigated by TEM. Cross-sectional TEM images of the $\mathrm{GaN}-\mathrm{Au}$ interfaces formed on the surface annealed at $600{ }^{\circ} \mathrm{C}$ and on the surface cleaned by the Ga reflux method are presented on Fig. 9. The HF etched interface could not be examined because of a poor $\mathrm{Au}$ adherence, which made TEM sample preparation impossible. The annealed surface results in a well-defined, abrupt $\mathrm{Au}-\mathrm{GaN}$ interface, in good agreement with the XPS and $I-V$ data for this interface. A more detailed TEM study of this interface has been published elsewhere. ${ }^{23}$

Conversely, the $\mathrm{Ga}$ reflux interface presents a much more complex structure, with evidence of $\mathrm{Au}-\mathrm{GaN}$ intermixing. Figure 9(b) shows the presence of an intermediate layer about $600 \AA$ thick between GaN and polycrystalline Au. This layer consists of $\mathrm{Au}$ grains embedded in a $\mathrm{GaN}$ matrix, as show by the magnified regions. This is in good agreement with the evolution of $\mathrm{Au} 4 f_{7 / 2}$ core-level binding energy which indicates the presence of $\mathrm{Ga}-\mathrm{Au}$ alloying within a thicker interfacial layer than for the HF etched and annealed surfaces. The complex interface could also explain the MFP anomaly discussed earlier, as well as the poor diodic properties.

\section{DISCUSSION}

Analysis of the results revealed striking differences in the interface formation mechanisms and electrical properties 

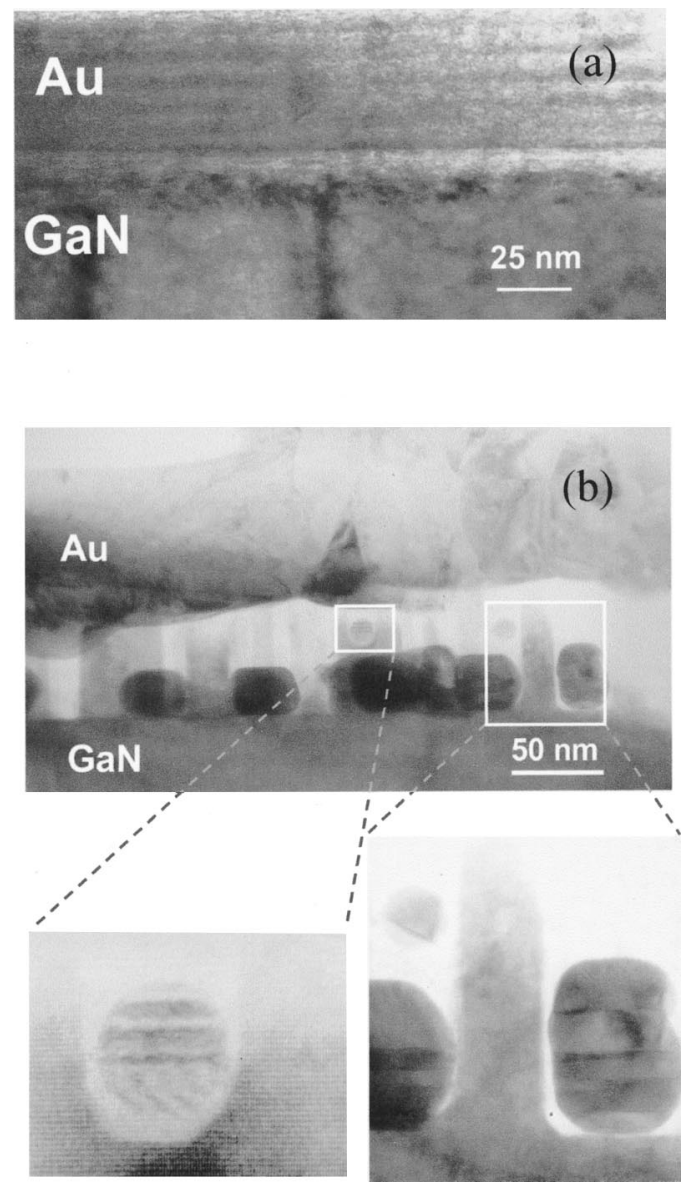

FIG. 9. Cross-section micrograph of the $\mathrm{Au}-\mathrm{GaN}$ interface formed after (a) an in situ $600{ }^{\circ} \mathrm{C}$ anneal and (b) GaN cleaning by the Ga reflux method.

of the contacts. It is important to stress that the three samples used in the study have been cut from the same wafer and subjected to the same HF etch, so that the number of variable parameters have been kept to a minimum. This is particularly important in the case of $\mathrm{GaN}$, as growth techniques are not as well established as for other semiconductor materials.

The diodes formed on the $600{ }^{\circ} \mathrm{C}$ annealed surface yielded an average barrier height of $1.24 \mathrm{eV}$, compared to $0.84 \mathrm{eV}$ for the Ga reflux surface. The total XPS band bending for the Ga reflux surface $(0.45 \mathrm{eV})$ is less than that of the $600^{\circ} \mathrm{C}$ annealed surface $(0.6 \mathrm{eV})$, which is consistent with the barrier heights measured by $I-V$. However, the difference in mean barrier height $(0.4 \mathrm{eV})$ is greater than the band bending difference $(0.15 \mathrm{eV})$. This could be a consequence of the poor uniformity and high-ideality factors, which might lead to underestimation of the barrier height. Indeed, when the interface presents local variation of the barrier height, as seems to be the case for the Ga reflux surface, the $I-V$ technique tends to yield the lowest value while XPS gives a weighted average of the band bending.

The fact that the total XPS band bending $(0.6 \mathrm{eV})$ for the $600{ }^{\circ} \mathrm{C}$ annealed surface is much less than the barrier height measured by $I-V(1.24 \mathrm{eV})$ suggests that the HF etched surface presents an initial band bending of about $0.64 \mathrm{eV}$.

Bermudez $^{24}$ has suggested that the behavior of metalGaN contacts could be interpreted within the Cowley-Sze model, ${ }^{25}$ where the barrier height is expressed as a sum of the "bare surface barrier height" and a Schottky-Mott term. This model assumes a uniform distribution of surface states, the density of which, in the case of $\mathrm{GaN},{ }^{24}$ is strongly dependent on surface preparation.

Wu et al. ${ }^{10}$ and Sporken et al. ${ }^{9}$ have, respectively, reported a bare surface-barrier height of 0.7 and $2.2 \mathrm{eV}$ and an Au-induced band bending of $0.5 \mathrm{eV}$ and $-1.05 \mathrm{eV}$, as measured by photoemission. The final barrier heights are similar $(1.2 \mathrm{eV})$ and compare well with our results, though the mechanisms of interface formation are different. The Cowley-Sze model cannot account for Sporken's result, since $\mathrm{Au}$ deposition caused the bands to bend in the opposite way to that predicted by the Schottky-Mott theory.

According to the Cowley-Sze model, the bare surface barrier height at the $600{ }^{\circ} \mathrm{C}$ annealed surface would be about $1.0 \mathrm{eV}$ and the Schottky-Mott contribution $0.25 \mathrm{eV}$. Assuming a value of $3.3 \mathrm{eV}$ for the $\mathrm{GaN}$ electron affinity, ${ }^{10,24}$ this leads to a density of surface states of about 2.3 $\times 10^{13} \mathrm{~cm}^{-2} \mathrm{eV}^{-1}$. ${ }^{24}$ This high density of surface states reflects the fact that most of the final barrier height is due to initial band bending, which occurs prior to metal deposition. Similarly, the Schottky-Mott contribution for the HF etched surface would be $0.6 \mathrm{eV}$, indicative of a lesser degree of Fermi-level pinning than for the annealed surface, with a bare surface barrier height of $0.65 \mathrm{eV}$.

The anneal therefore caused an increase in the density of surface states ${ }^{18}$ which enhanced band bending at the surface, from $0.65 \mathrm{eV}$ for the HF etched surface to $1.0 \mathrm{eV}$. The initial band bending at the HF etched surface is somewhat unexpected as practical surfaces are thought to display near flatband condition. ${ }^{26}$ One possible explanation is that the surface states responsible for this band bending might be caused by structural defects created during $\mathrm{GaN}$ growth, such as threading dislocations spreading to the surface. The low mobility and carrier concentration of the layer does indicate a highdefect density, ${ }^{27}$ which might explain the high-final Schottky barrier. Even though the annealing temperature used was approximately $400^{\circ} \mathrm{C}$ lower than the temperature at which the $\mathrm{GaN}$ was grown, there is also a possibility that the annealing process (in UHV, as opposed to nitrogen overpressure) itself induces or activates defects at the GaN surface. ${ }^{20,21}$ To explore the possible significance of this effect would require a detailed structural examination of $\mathrm{GaN}$ as function of annealing temperature and will be the subject of a future study.

Spicer et al. ${ }^{28}$ suggested that Schottky barrier heights are nearly always determined by defects at the interface. In the so-called "unified defect model," it is assumed that defects are generated near the semiconductor surface when the metal contact is deposited on that surface. These defects in turn lead to Fermi-level pinning. According to this model, our results for the etched and annealed surfaces could be interpreted as follows:

(a) Au deposition on the HF etched surface induces defects and causes a 0.6 upward band bending.

(b) Annealing at $600{ }^{\circ} \mathrm{C}$ induces defects and an upward band bending of $0.35 \mathrm{eV}$, then $\mathrm{Au}$ deposition pulls the bands a further $0.25 \mathrm{eV}$ up by creating more defects. 
The Ga reflux surface resulted in a more complex interface, which cannot be described fully by the Cowley-Sze model. The initial Au depositions induce upward band bending and could be interpreted in terms of the Schottky-Mott model, but the downward band bending at higher Au coverage calls for a different interpretation. Downward band bending has been observed by Sporken et al. ${ }^{9}$ They found that their XPS measured Au- $n$ GaN Schottky barrier height (1.15 $\mathrm{eV}$ ) was in good agreement with that predicted by the metal induced gap states (MIGS) and electronegativity model ${ }^{29,30}$ $(0.96 \mathrm{eV})$. Our $I-V$ measured barrier height $(0.84 \mathrm{eV})$ is also in good agreement with this model. However, the MIGS model does not take into account reacted interfaces.

The effective work function model ${ }^{31}$ was found to give good predictions for a number of reacted Au contacts to III-V and II-VI semiconductor compounds. This model states that chemical reactions during metal deposition tend to generate excess anions at the interface and therefore the Schottky barrier height could be given by the difference of the anion work function and the electron affinity. In practice, however, this model is not applicable to contacts to $\mathrm{GaN}$ since the work function of $\mathrm{N}$ is not defined.

We have seen previously that both surfaces are likely to present a high density of defects, which usually results in tunneling and recombination currents. However, these defects do not seem to be electrically active in the case of the $600{ }^{\circ} \mathrm{C}$ annealed surface, as indicated by the low ideality factors and good uniformity of the contacts. This changes drastically when the surface is cleaned by the Ga reflux technique prior to metallization. Similar behavior was observed for $\mathrm{Ag}-\mathrm{GaN}$ diodes fabricated on $n \mathrm{GaN}$ after $\mathrm{Ga}$ reflux cleaning. ${ }^{32}$ It is possible that the cleaning technique activated the defects and/or created additional, electrically active defects, since annealing at high temperature has been shown to induce defect states within the band gap. ${ }^{20,21}$

It is also possible that the high-temperature annealing steps involved in the Ga reflux cleaning process resulted in loss of $\mathrm{N}$ at the surface, ${ }^{33}$ which might not necessarily be detected by XPS, because of the relatively low sensitivity $(0.1 \%)$. As $\mathrm{N}$ vacancies act as donors, ${ }^{34}$ this loss of $\mathrm{N}$ would render the surface highly $n$-type, therefore enhancing tunneling.

Another possible explanation lies with the oxygen content at the surface. Surface oxygen has been reported to diffuse along threading dislocations to form a $\mathrm{GaO}$ layer underneath the interface. The yellow luminescence band observed in molecular-beam epitaxy grown $\mathrm{GaN}$ has been linked to this $\mathrm{GaO}$ layer. It is therefore possible that the surface defects could be affected by the $\mathrm{GaO}$ layer and perhaps neutralized in the case of the annealed surface, but not for the $\mathrm{Ga}$ reflux surface as it contains less oxygen. Depth profiling by XPS or Auger electron spectroscopy would reveal more information on this question and will be the subject of a future study.

\section{CONCLUSION}

Premetallization surface treatment was found to play a very important role in the structural and electrical properties of $\mathrm{Au}-n \mathrm{GaN}$ contacts. XPS and TEM studies showed that $\mathrm{Au}$ contacts formed after an in situ $600{ }^{\circ} \mathrm{C}$ anneal exhibited a smooth, abrupt interface while contacts formed after in situ cleaning by the Ga reflux method displayed a reacted, more complex interface, with evidence of $\mathrm{Au}-\mathrm{GaN}$ mixing. Analysis of the XPS core-level binding-energy position showed that both surface treatments caused upward band bending at the surface. The first few Au depositions induced further upward band bending in both cases, but the Ga reflux surface underwent reverse band bending as the Au coverage increased while the $600{ }^{\circ} \mathrm{C}$ annealed surface did not change. The total band bending induced by Au deposition was 0.25 and $0.0 \mathrm{eV}$ for the $600{ }^{\circ} \mathrm{C}$ annealed surface and Ga reflux, respectively.

Transport measurements performed on the same samples after processing confirmed the fundamental difference between the two surfaces. Contacts formed on the $600^{\circ} \mathrm{C}$ annealed surface and $\mathrm{Ga}$ reflux surface exhibited average Schottky barrier heights of 1.24 and $0.84 \mathrm{eV}$, respectively. The lowest ideality factors were 1.03 and 1.18 , respectively. The contact uniformity was very good in the case of the annealed surface and poor for the Ga reflux surface, in good agreement with XPS and TEM studies.

XPS study of Au deposition on the third surface, which was only subjected to an ex situ cleaning in a HF:DI $\mathrm{H}_{2} \mathrm{O}$ solution, showed a similar behavior to the $600^{\circ} \mathrm{C}$ annealed surface. The total band bending induced by the Au deposition was $0.6 \mathrm{eV}$, which is equal to the sum of the band bending caused by the $600{ }^{\circ} \mathrm{C}$ anneal and the subsequent Au deposition.

The Cowley-Sze and unified defect models have been put forward to interpret the behavior of the HF etched and annealed surfaces and suggest a high density of acceptor states at the surface. The high barrier reported is possibly a consequence of this high density of states, themselves caused by a high density of structural defects. These defects did not seem to act as recombination or tunneling centers for the $600^{\circ} \mathrm{C}$ annealed surface as opposed to the Ga reflux surface, whose electrical behavior suggests electrically active defects. The complexity of the interface in the latter case means that no single model of Schottky barrier formation can interpret the results in a satisfactory manner.

In conclusion, we have reported what we believe to be the highest barrier, lowest ideality factor $\mathrm{Au}-\mathrm{GaN}$ Schottky contacts, which exhibit excellent uniformity over a large number of diodes. This has been achieved though the relatively straightforward step of depositing Au in situ following a high-vacuum $\mathrm{GaN}$ annealing process. Our results indicate that this may be an appropriate processing stage in the production of nitride-based field-effect transistors.

\footnotetext{
${ }^{1}$ A. C. Schmitz, A. T. Ping, M. Asif Khan, Q. Chen, J. W. Yang, and I. Adedisa, J. Electron. Mater. 27(4), 255 (1998).

${ }^{2}$ Z. Z. Bandić, P. M. Bridger, E. C. Piquette, T. C. McGill, R. P. Vaudo, V. M. Phanse, and J. M. Redwing, Appl. Phys. Lett. 74(9), 1266 (1999).

${ }^{3}$ P. Hacke, T. Detchprom, K. Hiramatsu, and N. Sakawi, Appl. Phys. Lett. 63(19), 2676 (1993).

${ }^{4}$ F. D. Auret, S. A. Goodman, F. K. Koschnick, J-M. Spaeth, B. Beaumont, and P. Gibart, MRS Internet J. Nitride Semicond. Res. 4S1, G6.13 (1999).

${ }^{5}$ M. R. H. Khan, T. Detchprom, P. Hacke, K. Hiramatsu, and N. Sawaki,
} 
Transactions 2nd International High Temperature Electrical Conference, Charlotte, NC, 2, P-231 (1994).

${ }^{6}$ L. He, Z. J. Wang, and R. Zhang, J. Vac. Sci. Technol. A 17(4), 1217 (1999).

${ }^{7}$ E. V. Kalinia, N. I. Kuznetsov, V. A. Dmitriev, K. G. Irvine, and C. H. Carter, Jr., J. Electron. Mater. 25(5), 831 (1996).

${ }^{8}$ Y. Koyama, T. Hashizume, and H. Hasegawa, Solid-State Electron. 43, 1483 (1999).

${ }^{9}$ R. Sporken, C. Silien, F. Malengrau, K. Grigorov, R. Caudano, F. J. Sánchez, E. Cajella, E. Munoz, B. Beaumont, and P. Gibart, MRS Internet J. Nitride Semicond. Res. 2, 23 (1997).

${ }^{10}$ C. I. Wu and A. Kahn, J. Vac. Sci. Technol. B 16(4), 2218 (1998).

${ }^{11}$ M. Asif Khan, J. N. Kuznia, D. T. Olson, and R. Kaplan, J. Appl. Phys. 73(6), 3108 (1993).

${ }^{12}$ V. M. Bermudez, R. Kaplan, M. A. Khan, and J. N. Kuznia, Phys. Rev. B 48(4), 2436 (1993).

${ }^{13}$ T. U. Kampen and W. Mönch, Appl. Surf. Sci. 117/118, 388 (1997).

${ }^{14}$ S. C. Binari, H. B. Dietriech, G. Kelner, L. B. Rowland, K. Doverspike, and D. C. Gaskill, Electron. Lett. 30(11), 909 (1994).

${ }^{15}$ T. G. G. Maffeis, M. C. Simmonds, F. Peiro, P. Haines, P. J. Parbrook, and S. A. Clark, J. Phys. D 33(20), L115 (2000).

${ }^{16}$ M. P. Seah and W. A. Dench, Surf. Interface Anal. 1, 2 (1979).

${ }^{17}$ R. Ludeke, Surf. Sci. 132(1-3), 143 (1983).

${ }^{18}$ V. M. Bermudez, D. D. Koleske, and A. E. Wickenden, Appl. Surf. Sci. 126(1-2), 68 (1998).

${ }^{19}$ D. T. Jayne, N. S. Fatemi, and V. G. Weizer, J. Vac. Sci. Technol. A 10(4), 2802 (1992).
${ }^{20}$ L. J. Brillson, T. M. Levin, G. H. Jessen, A. P. Young, C. Tu, Y. Naoi, F. A. Ponce, Y. Yang, G. J. Lapeyre, J. D. MacKenzie, and C. R. Abernathy, Physica B 273-274, 70 (1999).

${ }^{21}$ F. D. Auret, S. A. Goodman, F. K. Koschnick, J-M. Spaeth, B. Beaumont, and P. Gibart, MRS Internet J. Nitride Semicond. Res. 4S1, G6.13 (1999).

${ }^{22}$ J. H. Werner, Appl. Phys. A: Mater. Sci. Process. 47, 291 (1988).

${ }^{23}$ F. Peiro, A. Cornet, T. G. G. Maffeis, M. C. Simmonds, and S. A. Clark, Microscopy, Barcelona, 2001.

${ }^{24}$ V. M. Bermudez, J. Appl. Phys. 86(2), 1170 (1999).

${ }^{25}$ A. M. Cowley and S. M. Sze, J. Appl. Phys. 36, 3212 (1965).

${ }^{26}$ V. M. Bermudez, T. M. Jung, K. Doverspike, and A. E. Wickenden, J. Appl. Phys. 79(1), 110 (1996).

${ }^{27}$ C. Y. Hwang, M. J. Schurman, W. E. Mayo, Y. C. Lu, R. A. Stall, and T. Salagaj, J. Electron. Mater. 26(3), 243 (1997).

${ }^{28}$ W. E. Spicer, I. Lindau, P. Skeath, C. Y. Su, and P. W. Chye, Phys. Rev. Lett. 44, 420 (1980).

${ }^{29}$ V. Heine, Phys. Rev. A 138, 1689 (1965).

${ }^{30}$ W. Mönch, J. Vac. Sci. Technol. B 17(4), 1867 (1999).

${ }^{31}$ J. L. Freeouf and J. M. Woodall, Appl. Phys. Lett. 39(9), 727 (1981).

${ }^{32}$ T. G. G. Maffeis, S. A. Clark, P. R. Dunstan, S. P. Wilks, D. A. Evans, F. Peiro, and P. Parbrook, Phys. Status Solidi A 176, 751 (1999).

${ }^{33}$ S. W. King, J. P. Barnak, M. D. Bremser, K. M. Tracy, C. Ronning, R. F. Davis, and R. J. Nemanich, J. Appl. Phys. 84(9), 5248 (1998).

${ }^{34}$ S. Nakamura and G. Fasol, The Blue Laser Diode (Springer, New York, 1997). 